\title{
A Design Method of Cognitive Overlay Links for Satellite Communications
}

\author{
Luciano Barros Cardoso da Silva \\ IMT Atlantique, Lab-STICC, Toulouse \\ luciano.barroscardosodasilva@imt-atlantique.fr
}

\author{
Tarik Benaddi \\ IMT Atlantique, Lab-STICC, Toulouse \\ tarik.benaddi@imt-atlantique.fr
}

\author{
Laurent Franck \\ Airbus Defence and Space \\ laurent.franck@airbus.com
}

\begin{abstract}
In this paper, we present a method to design cognitive overlay links for satellite communications, in order to allow the primary and the cognitive users to transmit concurrently while using efficiently the available power resources. By means of trellis shaping based dirty paper coding (DPC) and superposition techniques, numerous schemes are investigated in various realistic scenarios, and different tradeoffs between power efficiency vs complexity are made. By simulations, we first show that we are able to design schemes where the primary user bit error rate (BER) is maintained as in the absence of the cognitive user interference. Secondly, thanks to trellis precoding and an appropriate constellation expansion, we show that the BER of the cognitive user can be made within $1 \mathrm{~dB}$ of corresponding the Gaussian channel BER.
\end{abstract}

Index Terms-Satellite Communications, Cognitive Radio, Overlay Paradigm, Dirty Paper Coding, Trellis Precoding, Flexible Payloads

\section{INTRODUCTION}

In the past few years, the demand for new rising satellite services in respect to global connectivity, broadcast capabilities and mobility aspects has experienced a rapid growth [1]. For instance, we point out the use of satellite for the support of machine-to-machine (M2M) communications, providing reliable services where terrestrial systems are inaccessible or even unaffordable. Recent projections highlight a deployment of around 1 million device $/ \mathrm{km}^{2}$ in the next couple of years (smart sensors, vehicles, healthcare monitoring ... ) [2]. By contrast, despite their advantages when compared to terrestrial networks, the satellite systems also face unique design challenges, such as: (i) low power consumption, (ii) the use, whenever possible, of commercial off-the-shelf (COTS) parts, which might limits the onboard processing resources, (iii) the development of technical solutions for enhancing spectrum coexistence among different users, in order to alleviate the increasingly spectral scarcity.

It is within this framework that the cognitive radio (CR) techniques have also emerged for satellite communications [3], benefiting from the technical advances in flexible payloads [4] [5] and software defined radio technology [6]. On this subject, the recent works [7], [8] and [9] presented contributions to interweave and underlay $\mathrm{CR}$ paradigms for satellite communication systems, more specifically with respect to satellite-terrestrial integrated systems and dual satellite systems. In a nutshell, the interweave paradigm is defined by an opportunistic transmission of the unlicensed cognitive user (CU) by sensing the licensed primary user (PU) presence and searching for a gap in frequency, time or polarization. Conversely, in the underlay paradigm the CU transmits simultaneously with the PU, while respecting to the power threshold specified by an interference temperature sensing method.

With regard to the above contributions, this paper in turn proposes the use of overlay CR paradigm for satellite communications. This approach is well suitable for the aforementioned context, especially due to the fact that the satellite has a complete and noncausal knowledge of all users messages prior to the transmission, a key requirement for the overlay paradigm. In this sense, both PU and CU are able to transmit concurrently at the same frequency, time and space. The main advantage of this scheme is that the infrastructure of the PU communication system remains unchanged as well as its performance. On the other hand, the CU adapts its transmission, by means of advanced encoding strategies, to mitigate the PU interference in the cognitive link and to maintain the signal to noise ratio of the primary link.

In accordance with the interference model presented in [10], the optimal CU encoding strategy for overlay CR paradigm consists of two independent strategies: superposition and dirty paper coding (DPC) [11]. In the superposition technique, the CU shares part of its power in order to relay the PU transmission. As a result, considering the proper amount of shared power, the signal-to-interferenceplus-noise ratio (SINR) at the PU receiver remains constant, as if the interference were absent. Subsequently, with the remaining available power after superposition, a trellis shaping based DPC scheme, similar to [12] and [13], is implemented to presubtracted the known PU interference before transmission.

Based on the foregoing techniques, this work presents a method to control the CU transmitted power in order to ensure constant SINR at PU receiver, in accordance with the superposition strategy. In this way, the $\mathrm{CU}$ power resource is efficiently utilized - an important requirement for satellite communications. We propose to evaluate the shaping gain and use it as a scaling factor for the CU constellation minimum distance. Moreover, depending on the strength of the interference, further constellation expansion as well as modulo quantization are considered. Different configurations and scenarios are simulated and analyzed, leading to design trade-offs between power efficiency and complexity.

Following this introduction, the section II presents a system description and the general concepts of superposition and DPC techniques. Subsequently, in Section III, the system design is exposed. Simulation and results are discussed in Section IV. Finally, the Section V is dedicated to the conclusions with suggestions for future works. 


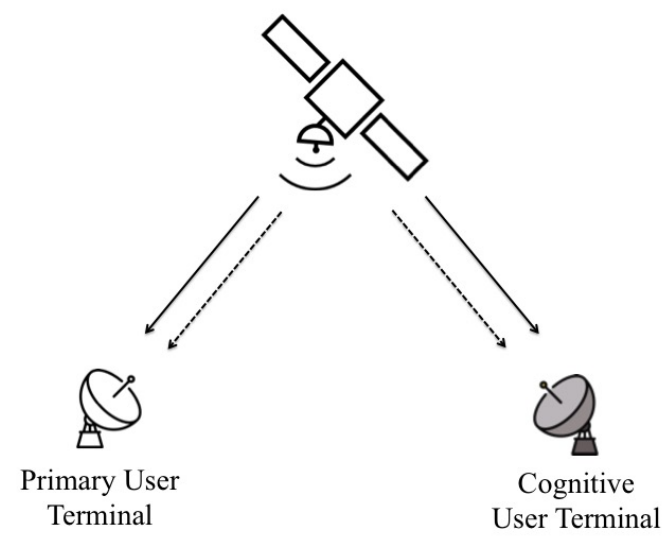

(a) LEO/MEO satellite scenario

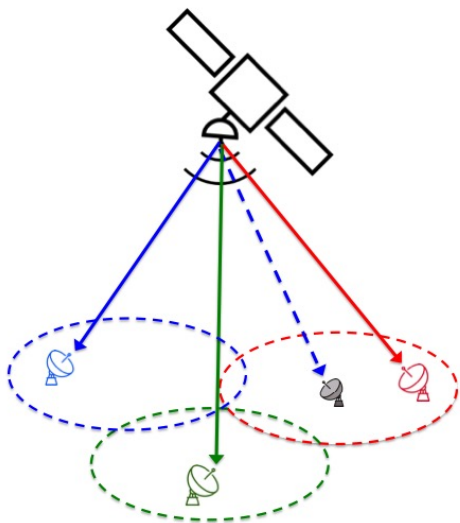

(b) Multibeam satellite scenario

Fig. 1: Examples of Satellite Scenarios: PU - solid line; CU - dashed line

\section{System Description}

\section{A. Overlay Model and Superposition Strategy}

The main motivation to introduce the overlay $\mathrm{CR}$ technique for satellite communications lies in the feasibility of transmitting both unlicensed and licensed services simultaneously towards its respective terminals. We point out that the difference in respect to the broadcast channel is the priority among users, which requires additional requirements and countermeasures.

The Fig. 1a and Fig. 1b present examples of scenarios where the overlay technique might be applied. The first one presents a LEO/MEO satellite, where two different services are provided. The PU is the licensed one and takes priority over the CU. In the second example, a GEO multibeam satellite transmits both licensed and unlicensed services, utilizing, for instance, flexible payloads techniques. In this case, the cognitive user signal is transmitted in the red frequency area but using the blue frequency, in order to avoid interference with the ground terminals. Hence, because of this frequency reuse, the interference among adjacent beams shall be controlled.

In both scenarios, the standard form of the interference model with side information presented in Fig. 2 can be applied. The primary and cognitive signals are onboard the satellite. Under this assumption, the cognitive encoder has full and noncausal knowledge of the PU signal. Thus, the encoded cognitive signal $X_{c}^{n}$ is function of both primary and cognitive messages $m_{p}$ and $m_{c}$, respectively. On the other hand, the PU signal $X_{p}^{n}$ encoding strategy remains the same. Without loss of generality, the cognitive channel gains are defined by $(1, a, b, 1)$, with direct paths assuming unitary gains and the gains of the interfering paths defined as $a, b \in[0,1]$. The following equations describes the channel:

$$
\begin{aligned}
& Y_{p}^{n}=X_{p}^{n}+a X_{c}^{n}+Z_{p}^{n} \\
& Y_{s}^{n}=X_{c}^{n}+b X_{p}^{n}+Z_{s}^{n},
\end{aligned}
$$

where the noise component $Z_{p}^{n}$ is assumed as $\mathcal{N}\left(0, N_{p}\right)$ and $Z_{s}^{n}$ as $\mathcal{N}\left(0, N_{s}\right)$. Also, the power constraints to be satisfied by the primary and cognitive users are $E\left[\left|X_{p}^{n}\right|^{2}\right]=$ $P_{p}$ and $E\left[\left|X_{c}^{n}\right|^{2}\right] \leq P_{c}$, respectively.

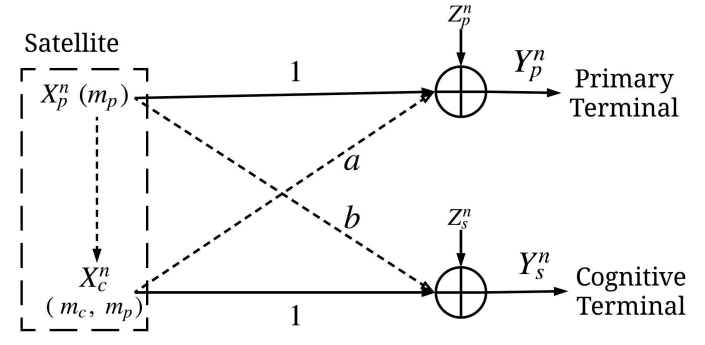

Fig. 2: Channel Model

Given the channel model, the first condition to be accomplished is to ensure the PU performance in the presence of $\mathrm{CU}$ transmission. To fulfill this requirement, the CU signal shares part of its power to relay the PU, in order for this later to overcome the additional $\mathrm{CU}$ interference. Based on that operation, the CU transmitted signal is given by:

$$
X_{c}^{n}=\hat{X}_{c}^{n}+\sqrt{\alpha \frac{P_{c}}{P_{p}}} X_{p}^{n},
$$

where $\alpha \in[0,1]$ is the shared fraction of power $P_{c}$.

It shall be noticed that a new power constraint $E\left[\left|\hat{X}_{c}^{n}\right|^{2}\right] \leq(1-\alpha) P_{c}$ is defined, assuming that the components of Eq. (3) are statistically independents. By rearranging Eq. (1), the signal-to-interference-plus-noise ratio (SINR) at the primary receiver is given by:

$$
S I N R_{P}=\frac{E\left[\left|\left(1+a \sqrt{\alpha^{*} \frac{P_{c}}{P_{p}}}\right) X_{p}^{n}\right|^{2}\right]}{E\left[\left|a \hat{X}_{c}^{n}\right|^{2}\right]+E\left[\left|Z_{p}^{n}\right|^{2}\right]},
$$

Without loss of generality, we assume the noise power $N_{s}$ at PU receiver as unitary. Under the condition of statistical independence among the components in Eq. (3), the superposition factor $\alpha^{*} \in[0,1]$ that maximizes the PU SINR, for interference condition $(a>0)$, is defined by [10]:

$$
\alpha^{*}=\left(\frac{\sqrt{P_{p}}\left(\sqrt{1+a^{2}\left(1+P_{p}\right) P_{c}}-1\right)}{a \sqrt{P_{c}}\left(1+P_{p}\right)}\right)^{2} .
$$

It is worth noting from Eq. (4) that, in accordance with interference channel model, despite of the demodulator at 
the PU terminal receives the specified SINR, the received signal power is increased by the superposition component $\left(a \sqrt{\alpha^{*} \frac{P_{c}}{P_{p}}}\right) X_{p}^{n}$. In this paper, emphasizing the practical aspects, we assumed that the PU infrastructure is unchanged.

\section{B. Dirty Paper Coding}

\section{1- Transmitter side}

After the superposition, the objective is to design the signal $\hat{X}_{c}^{n}$ in such way to mitigate the PU interference. By means of the theoretical results presented by Costa [11], on the assumption that the interference is noncausally known at transmitter, a transmitter-based interference presubtraction can be implemented without any power increase, reaching the AWGN capacity.

Having regard to the practical implementations of DPC, Erez, Shamai and Zamir introduced the pioneer work concerning this subject [14], pointing out the use of Tomlinson-Harashima precoding (THP) for intersymbol interference (ISI) cancelling. In addition, also for Gaussian ISI channels, Eyuboglu and Forney in [15] presented the trellis precoded (TP), which combines the trellis shaping (TS) technique [16] with THP. In this latter scheme, besides the interference mitigation, the shaping loss could be partially recovered. Similarly with our application, an extension of the TP technique for multiuser interference was proposed in [12] and further explored in [13].

By rearranging the Eq. (2) and considering the superposition, the received signal is given by:

$$
Y_{s}^{n}=\hat{X}_{c}^{n}+\underbrace{\left(b+\sqrt{\alpha \frac{P_{c}}{P_{p}}}\right) X_{p}^{n}}_{S^{n}}+Z_{s}^{n},
$$

where $S^{n}$ is the transmitter known channel interference.

Through the lattice strategy described in [14], it was observed that the partial interference pre-subtraction (PIP) outperforms the total interference pre-subtraction operating at low or intermediate signal-to-noise ratio regimes. In accordance with this assumption, the signal $\hat{X}_{c}^{n}$ is designed as:

$$
\hat{X}_{c}^{n}=\left[X_{c c}^{n}-\lambda S^{n}\right] M O D_{\Delta},
$$

where $X_{c c}^{n}$ is the coded signal and the factor $\lambda$, to be properly chosen, controls the fractioned interference to be presubtracted. Also, $M O D_{\Delta}$ is the complex-valued modulo operation. The amplitude is defined by $\Delta=\sqrt{M} d_{\min }$, where $M$ is the number of points of expanded square QAM constellation and $d_{\text {min }}$ the minimum intersymbol distance.

The modulo operation presubtracts the interference while assuring a low increasing power. However, its operation introduces some irreversible losses in the system, as characterized by [14]. For this reason, its implementation must be considered according to the interfering scenario. The equations presented in this paper take account of modulo operation in order to conserve the notation.

In some practical system, a dither is added at the transmitter and subtracted at the receiver in order to make the components of Eq. (7) statistically independents.

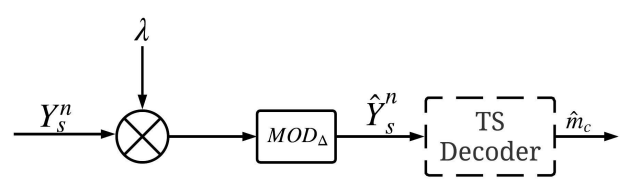

Fig. 3: DPC Receiver

\section{2- Receiver side}

The receiver operation, considering the elements presented in the last section, is illustrated by Fig. 3. At the decoder input, the signal is given by:

$$
\begin{gathered}
\hat{Y}_{s}^{n}=\left[\left(\hat{X}_{c}^{n}+S^{n}+Z^{n}\right) \lambda\right] M O D_{\Delta} \\
=\left[\lambda \hat{X}_{c}^{n}+\left(X_{c c}^{n}-\hat{X}_{c}^{n}\right) M O D_{\Delta}+\lambda Z_{s}^{n}\right] M O D_{\Delta} \\
=\left[X_{c c}^{n}-(1-\lambda) \hat{X}_{c}^{n}+\lambda Z_{s}^{n}\right] M O D_{\Delta},
\end{gathered}
$$

By Eq. (10), we define the effective noise for cognitive user link as $(1-\lambda) \hat{X}_{c}^{n}+\lambda Z_{s}^{n}$. As a consequence, the effective signal-to-noise ratio for cognitive user $S N R_{e f f}$ is given by:

$$
S N R_{e f f}=\frac{E\left[\left|X_{c c}^{n}\right|^{2}\right]}{E\left[\left|(1-\lambda) \hat{X}_{c}^{n}\right|^{2}\right]+E\left[\left|\lambda Z_{s}^{n}\right|^{2}\right]},
$$

The value of $\lambda$ that maximize Eq. (11) is given by [11]:

$$
\lambda=\frac{\left(1-\alpha^{*}\right) P_{c}}{\left(1-\alpha^{*}\right) P_{c}+E\left[\left|Z_{s}^{n}\right|^{2}\right]} .
$$

\section{System DESIGN}

\section{A. CU transmitted power}

From this point, the objective is to construct the coded signal $X_{c c}^{n}$ with a good channel coding gain and as close as possible to the interference $\lambda S^{n}$.

The Fig. 4 presents the implemented encoder for cognitive user. In essence, the upper part is formed by the coset select code $C_{c}$, specified by the generator matrix $G_{c}$, which encodes the $k_{c}$ bits message into a $n_{c}$ coded sequence. In parallel, in the lower part, the inverse syndrome former matrix $H_{s}^{-T}$, generated for the shaping code $C_{s}$, receives the $r_{s}$-bits syndrome sequence and provides at its output the initial shaping sequence $t$. Subsequently, by knowledge of $w, t$ and $\lambda S$, the Viterbi decoder selects the closest shaping codeword $y_{s}$ in respect to any metric chosen. The sequence $z_{s}$, formed by the XOR operation of $y_{s}$ and the original shaping sequence $t$ enters in the mapper to form, jointly with $w$, the shaped transmitted sequence $X_{c c}^{n}$. In the same way as presented in [16], this work utilized the trellis shaping on regions strategy for shaped mapping.

The following branch metric is implemented, where the precoder selects the proper region sequence with minimum average energy to steer the scaled interference sequence $\lambda S$.

$$
\left\|\left[X_{c c}^{n}-\lambda S^{n}\right] M O D_{\Delta}\right\|^{2}
$$

At the decoder, the operation is the same as implemented for the trellis coded modulation (TCM) decoder: 


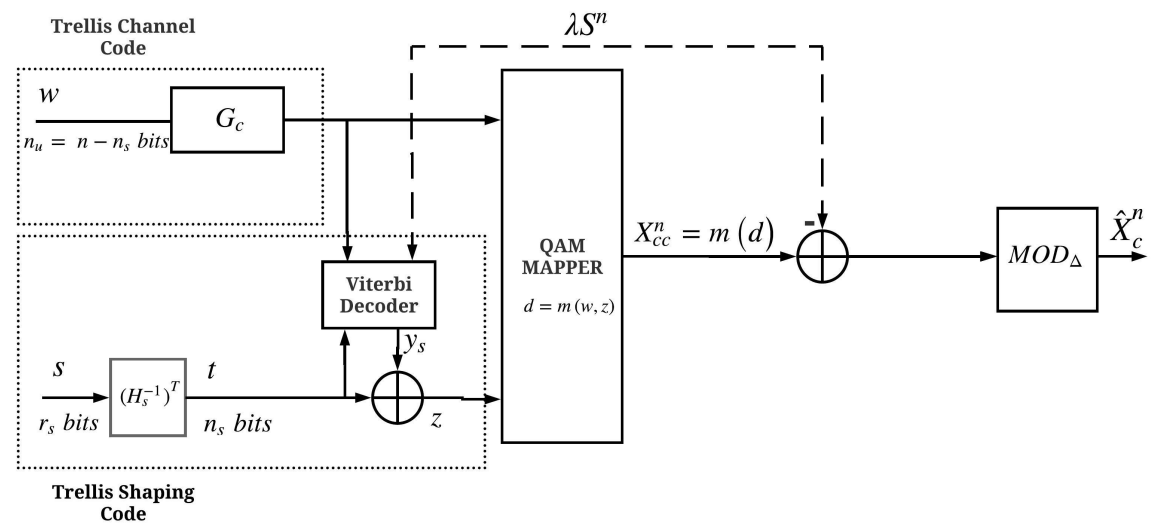

Fig. 4: Proposed DPC Encoder

the symbols are decoded using the expanded constellation mapping with a Viterbi decoder for $C_{c}$, where the trellis shaping represents the parallel branches between consecutive states transitions in the trellis. After that, the shaping bits are estimated by the syndrome mapper for $C_{s}$.

However, the possible power reduction of $\hat{X}_{c}^{n}$, caused by the trellis precoding technique, impacts directly on both links performances, since the value adopted for power sharing at the superposition strategy is no longer exact. It can be noted, by regarding the Eq. (4), that the SINR at primary receiver is increased and, as a result, the PU presents better bit error rate (BER) performance.

In this paper, we propose a method for controlling the CU output power such as $E\left[\left|X_{c}^{n}\right|^{2}\right]=P_{c}$, or equivalently, $E\left[\left|\hat{X}_{c}^{n}\right|^{2}\right]=(1-\alpha) P_{c}$. This is reached by proper scaling of the minimum distance $d_{\min }$ of the transmitted constellation.

According to the trellis precoding theory [15], the exactly same shaping gain generated by a shaping code $C_{s}$ is obtained for the multiuser precoding [12]. In line with this analysis, we define the power of the baseline, without considering the shaping operation, as:

$$
P_{\oplus}=\frac{2^{R}}{6} d_{m i n}^{2}
$$

where $R$ is the data rate in bits per two dimensions. For the proposed scheme, the shaping gain is defined as follows:

$$
\gamma_{s}=\frac{P_{\oplus}}{(1-\alpha) P_{c}} .
$$

Thus, the scaled minimum distance $d_{m i n}$, such that the available power after the shaping operation is equals to $(1-\alpha) P_{c}$, is defined by:

$$
d_{m i n} \prime=\sqrt{\frac{\left[(1-\alpha) P_{c}\right] 6 \gamma_{s}}{2^{R}}} .
$$

\section{B. Constellation Expansion}

When the interference is much higher than the signal constellation, the output power after the presubtraction is increased and the dirty paper technique can not be applied. In this case, two solutions can be implemented: either we add a modulo operation to confine the transmitted signal or, alternatively, we expand the CU constellation to get closer to the interference.

As an example, following the notation in Fig. 4, consider that the original DPC constellation is a 16-QAM, where each symbol is defined by the tupple $\left(z_{1} z_{2} c_{1} c_{2}\right)$ where $z_{1}$ and $z_{2}$ are the sign bits while $c_{1}$ and $c_{2}$ are the coset bits. This constellation can be expanded to a 64-QAM by considering an additionally two 'auxiliary' bits (not information bits) $z_{3}$ and $z_{4}$. Now, each symbol is defined by the tupple $\left(z_{1} z_{2} z_{3} z_{4} c_{1} c_{2}\right)$. That way, the $\mathrm{CU}$ constellation can be made arbitrarily larger, enough to confine the interference signal (here the PU symbols).

This operation could be seen as an extension of the trellis shaping procedure. The Viterbi decoder at transmitter acts as an usual TCM decoder, where the auxiliary shaping bits $z_{a u x}$ represent the parallel branches transitions between consecutive state transitions in the trellis for $C_{s}$.

\section{Simulation AND Results}

The PU is implemented as a 4-QAM signal encoded by a 16 -state, rate $1 / 2$, convolutional code $C_{c}$, specified in the octal notation by the generators $g_{1}(D)=31$ and $g_{2}(D)=33$. The transmitted power is variable according to the specific scenario.

For the $\mathrm{CU}$, in all simulated cases, the available transmitted power is assumed as $10 \mathrm{~W}$ and the transmission rate is $R_{c u}=2$ bits/symbol. The proposed DPC encoder presented in the Fig. 4 is implemented for some configurations.

In the upper part of the encoder, for all cases, the same systematic 64 -state, rate $1 / 2$, channel trellis convolutional code $C_{c}$, given by feedforward polynomial $h_{1}(D)=54$ and the feedback polynomial $h_{0}(D)=161$, is considered.

In the lower part of the encoder, for all cases, the same 4 -state, rate $1 / 2$, shaping code $C_{s}$, specified by generators $g_{s, 1}(D)=7$ and $g_{s, 2}(D)=5$ is assumed. We investigate the constellation expansion in 4 (with $n_{s}=2$ ) and 16 regions (with $n_{s}=4$, where 2 are auxiliary bits). It is important to point out that the minimum distance scaling method, previously exposed in Section III, was implemented in all simulated schemes. Additionally, the impact of modulo operation in the system is also discussed. 


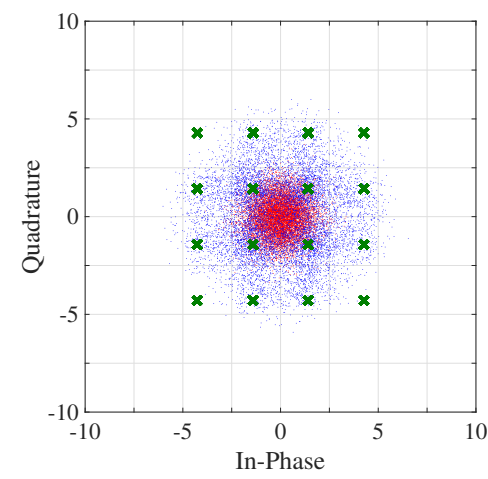

(a) $P_{P}=3 \mathrm{~dB}$

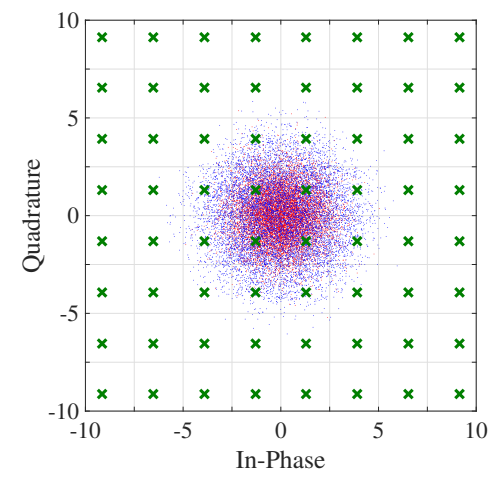

(b) $P_{P}=7 \mathrm{~dB}$

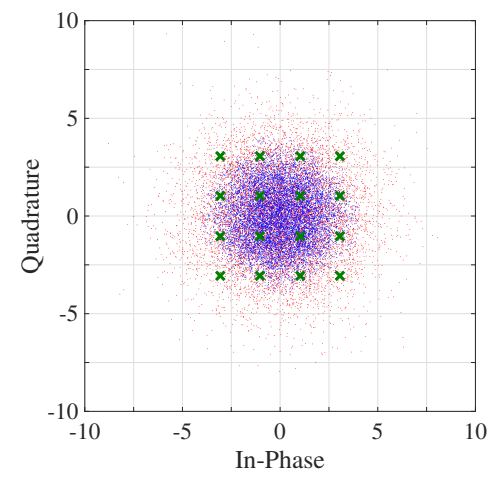

(c) $P_{P}=15 \mathrm{~dB}$

Fig. 5: Scatter plots of signal constellations at different PU interfering powers

In all cases, and without loss of generality, the interfering path gains $a$ and $b$ are assumed real values and equal to 0.2 , which represent the attenuation factors (antennas patterns, mismatches, earth station locations etc). The following scenarios, with respect of the PU power $P_{p}$, are evaluated at (i) $P_{p}=3 \mathrm{~dB}$; (ii) $P_{p}=7 \mathrm{~dB}$ and (iii) $P_{p}=15 \mathrm{~dB}$.

In other words, since the $P_{p}$ changes, a different value of the superposition factor $\alpha^{*}$ is computed at the CU design for each scenario, according to Eq. (5). As a consequence, the remaining power of the signal $\hat{X}_{c}^{n}$ as well as the amount of interference $S^{n}$ at CU receiver vary from case to case.

In order to analyze the system performance, the Fig. 5 presents the scatter plot of the signal constellations integrated in the partial interference pre-subtraction processing. The expanded constellation signal $X_{c c}^{n}$ is shown in green " $x$ ". The Gaussian distributed version of the scaled interference $\lambda S^{n}$ is superposed in red points and the transmitted signal $\hat{X}_{c}^{n}$ is shown in blue dots.

In the first scenario (i), for PU transmitted power $P_{p}$ of $3 \mathrm{~dB}$, the scheme with slight constellation expansion of 16 QAM has proved effective. The CU output power remains constant $(10 \mathrm{~W})$, which implies accurate superposition strategy. By the Fig. 5a, we notice that all interference is confined within the expanded constellation. In this case, we may infer that a significant part of shaping loss is recovered.

In such conditions, the scheme with more expansion, i.e. 64-QAM, is not be recommended, since the exactly same 16-QAM constellation points are going to be selected by the trellis shaping operation. Thus, the system complexity would be increased (more branches in the shaping code) without any effective gain.

The performance can be expressed, in a quantitative way, by the BER curves shown in Fig. 6. As expected, the scheme presented a gap less than $1 \mathrm{~dB}$ from the AWGN trellis shaping curve. In addition, we presented the same scheme with the modulo operation, coined as 16-QAM TP-M. It is clear that the modulo induces the so-called modulo losses in the system, as stated in [12] and [14].

Furthermore, in order to verify that PU operates properly, the simulated BER for PU at $P_{p} 3 \mathrm{~dB}$ is depicted in the presence and the absence of $\mathrm{CU}$ interference, i.e. 16-
QAM TP-M scheme. Thanks to the superposition and to the method of $d_{\text {min }}^{\prime}$ scaling, the BER PU, in the presence of $\mathrm{CU}$ interference, remains exactly the same as in the case of no CU interference.

In the second scenario (ii), for $P_{p}$ of $7 \mathrm{~dB}$, the interference can not be confined within the 16-QAM scheme. In this case, the CU output power is increased and the DPC condition is not satisfied. In Fig. 5b, we note that, by adopting the solution with 64-QAM, the interference remains inside of the expanded constellation region, which led us to expect that significant part of shaping loss could be recovered by the system.

The Fig. 7 presents the BER performance for this scenario. The results for 64-QAM scheme and 16-QAM TP-M are exposed. We observe that 64-QAM scheme performs within $1.5 \mathrm{~dB}$ to the AWGN trellis shaping BER curve. In addition, the 16-QAM TP-M scheme has a similar behaviour in relation to the previous scenario. Finally, as in the previous case, by using the 16-QAM TP-M signal as an interfering signal, the BER of the PU remains the same in comparison with the interference-free PU channel.

In the last scenario (iii), i.e. $P_{p}$ of $15 \mathrm{~dB}$, we can observe in Fig. 5c that a higher constellation expansion ( $n_{s}$ about 5 or 6 ) is necessary in order to accommodate the strong amount of interference. However, this expansion might not be reasonable for satellite communications due to the induced high complexity of the transmitter (here the satellite) and to the high 64-QAM peak-to-average ratio (PAPR). In this case, the 16-QAM TP-M should be adopted as depicted in Fig. 5c. Despite of the exact control of the CU output power, a near-uniform distribution of the transmitted signal is observed, which indicates that no shaping gain is reached. The Fig. 8 presents the BER curves for this scenario. We observe that the modulo loss is not significantly high when compared to the 64-QAM scheme.

\section{CONCLUSION}

This paper presents a design of cognitive overlay link for satellite communications. Different schemes were implemented in different realistic scenarios. The results presented the exactly same bit error rate for the primary user when compared with its performance on the corresponding AWGN channel. On the other hand, the cognitive user 


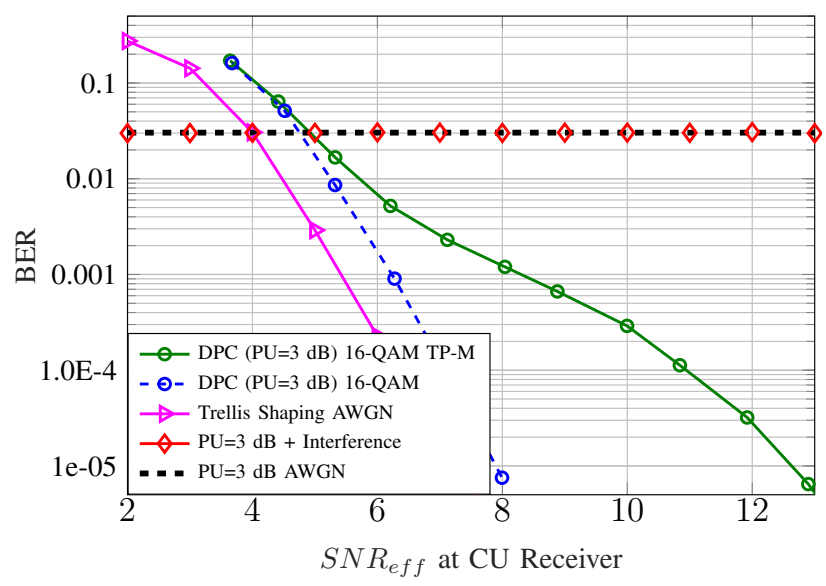

Fig. 6: BER of the Cognitive User at $P_{P}=3 \mathrm{~dB}$

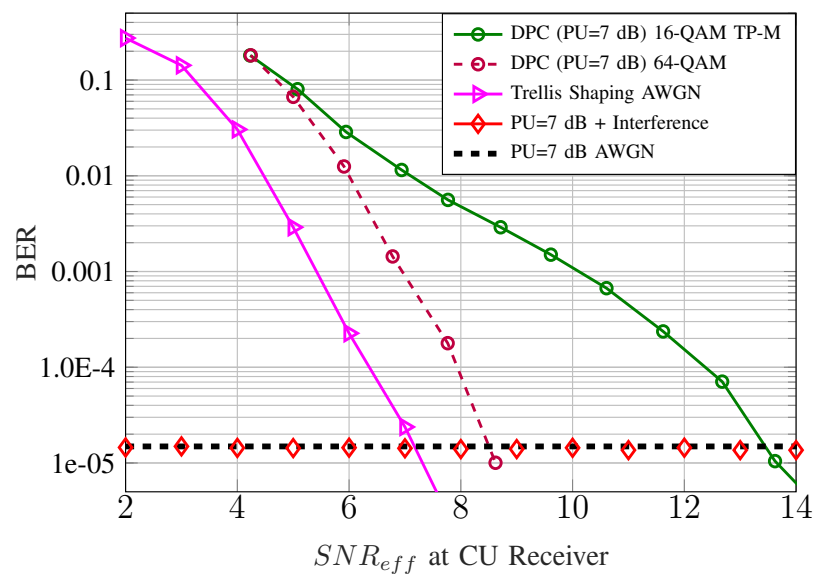

Fig. 7: BER of the Cognitive User at $P_{P}=7 \mathrm{~dB}$

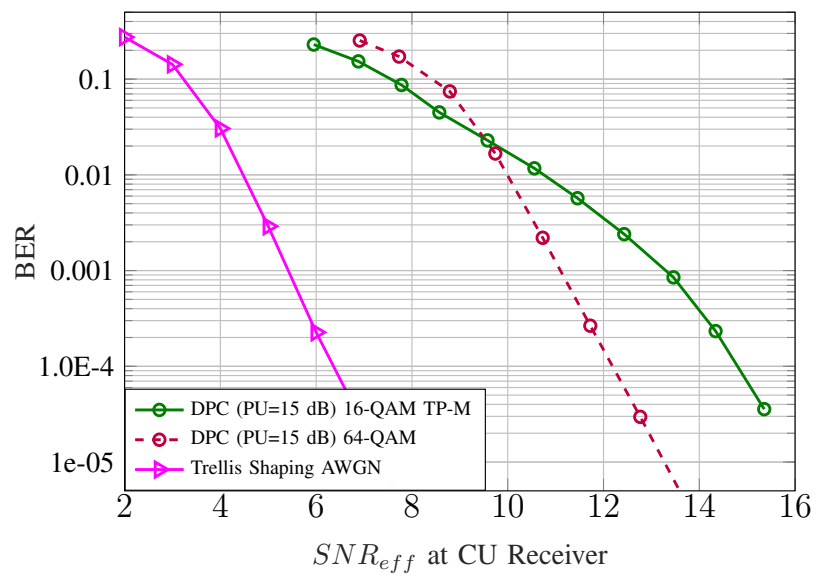

Fig. 8: BER of the Cognitive User at $P_{P}=15 \mathrm{~dB}$

presented a gap of around $1 \mathrm{~dB}$ in comparison to trellis shaping scheme over the AWGN channel.

In the future research, the typical impairments of satellite communications will be investigated for this transmission scheme. Moreover, we will analyze the overlay techniques considering nonlinear modulations.

\section{ACKNOWLEDGMENT}

This work was supported by National Council for Scientific and Technological Development (CNPq/Brazil) and by National Institute for Space Research (INPE/Brazil).

\section{REFERENCES}

[1] D. Minoli, Innovations in Satellite Communications and Satellite Technology: The Industry Implications of DVB-S2X, High Throughput Satellites, Ultra HD, M2M, and IP. John Wiley \& Sons, 2015.

[2] Z. Qu, G. Zhang, H. Cao, and J. Xie, "Leo satellite constellation for internet of things," IEEE Access, vol. 5, pp. 18 391-18401, 2017.

[3] K. Patil, K. Skouby, A. Chandra, and R. Prasad, "Spectrum occupancy statistics in the context of cognitive radio," in 14th International Symposium on Wireless Personal Multimedia Communications (WPMC), 2011. IEEE, 2011, pp. 1-5.

[4] G. Cocco, T. De Cola, M. Angelone, Z. Katona, and S. Erl, "Radio resource management optimization of flexible satellite payloads for dvb-s2 systems," IEEE Transactions on Broadcasting, 2017.

[5] N. Porecki, G. Thomas, A. Warburton, N. Wheatley, and N. Metzger, "Flexible payload technologies for optimising ka-band payloads to meet future business needs," in Proc. 19th Ka Broadband Commun., Navigat. Earth Observat. Conf., 2013, pp. 1-7.

[6] M. R. Maheshwarappa, M. Bowyer, and C. P. Bridges, "Software defined radio (sdr) architecture to support multi-satellite communications," in IEEE Aerospace Conference, 2015, pp. 1-10.

[7] S. K. Sharma, S. Chatzinotas, and B. Ottersten, "Cognitive radio techniques for satellite communication systems," in IEEE 78th Vehicular Technology Conference (VTC Fall), 2013, pp. 1-5.

[8] M. Höyhtyä, J. Kyröläinen, A. Hulkkonen, J. Ylitalo, and A. Roivainen, "Application of cognitive radio techniques to satellite communication," in IEEE International Symposium on Dynamic Spectrum Access Networks (DYSPAN), 2012. IEEE, 2012, pp. $540-551$.

[9] A. Vanelli-Coralli, A. Guidotti, D. Tarchi, S. Chatzinotas, S. Maleki, S. K. Sharma, N. Chuberre, B. Evans, M. LopezBenitez, W. Tang et al., "Cognitive radio scenarios for satellite communications: the corasat project," in Cooperative and Cognitive Satellite Systems. Elsevier, pp. 303-336.

[10] A. Jovicic and P. Viswanath, "Cognitive radio: An informationtheoretic perspective," IEEE Transactions on Information Theory, vol. 55, no. 9, pp. 3945-3958, 2009.

[11] M. Costa, "Writing on dirty paper (corresp.)," IEEE transactions on information theory, vol. 29, no. 3, pp. 439-441, 1983.

[12] W. Yu, D. P. Varodayan, and J. M. Cioffi, "Trellis and convolutional precoding for transmitter-based interference presubtraction," IEEE Transactions on Communications, vol. 53, no. 7, pp. 1220-1230.

[13] Y. Sun, W. Xu, and J. Lin, "Trellis shaping based dirty paper coding scheme for the overlay cognitive radio channel," in IEEE 25th Annual International Symposium on Personal, Indoor, and Mobile Radio Communication (PIMRC), 2014, pp. 1773-1777.

[14] U. Erez, S. Shamai, and R. Zamir, "Capacity and lattice strategies for canceling known interference," IEEE Transactions on Informa tion Theory, vol. 51, no. 11, pp. 3820-3833, 2005.

[15] M. V. Eyuboglu and G. D. Forney, "Trellis precoding: Combined coding, precoding and shaping for intersymbol interference channels," IEEE Transactions on Information Theory, vol. 38, no. 2, pp. 301-314, 1992.

[16] G. Forney, "Trellis shaping," IEEE Transactions on Information Theory, vol. 38, no. 2, pp. 281-300, 1992. 